\title{
An Efficient Smart Weighted and Neighborhood-enabled Load Balancing Scheme for Constraint Oriented Networks
}

\author{
Mohammed Amin Almaiah \\ Department of Computer Networks and Communications \\ College of Computer Sciences and Information Technology, King Faisal University, Saudi Arabia
}

\begin{abstract}
In Wireless Sensor Networks (WSNs), uniform load or traffic distribution strategy is one of the main challenging issues, which is tightly coupled with the resource-limited networks. To address this problem, various mechanisms have been developed and presented in the literature. However, these approaches were either application specific that is designed for a specific application area such as smart building or overlay complex. Therefore, a simplified and energy efficient loadbalancing scheme is always needed for the resource-limited networks. In this paper, an efficient and neighborhood-enabled load-balancing scheme is presented to resolve the aforementioned issues specifically with available resources. For this purpose, the proposed scheme bounds every member node to collect various information about neighboring nodes i.e., those nodes resides in its communication range. Moreover, if residual energy $\mathrm{Er}$ of sensor node is less than the defined threshold value then it shares this information with neighboring nodes. In the proposed neighborhood-enabled load balancing scheme, every sensor node $\mathrm{Ci}$ prefers to route packets through the optimal paths particularly those paths where probability of critical nodes is negligible i.e., path where critical node(s) are not deployed. Simulation results showed that the proposed neighborhoodenabled load-balancing scheme is better than existing approaches in terms of network lifetime (both individual node and whole WSNs), throughput, and average packet delivery ratio and endto-end delay performance metrics.
\end{abstract}

Keywords-Wireless Sensor Networks (WSNs); load balancing; PSO; routing protocol; low power devices

\section{INTRODUCTION}

With the development of wireless technologies, Wireless Sensor Networks (WSNs) must have low cost, low power consumption, small size, easy layout, and dynamically selforganized [1]. WSNs are using widely in many different applications, such as industrial applications, health care, military defense, environmental detection, target tracking, and ecological observation, etc. [2, 3]. However, because batteries supply the energy of the sensors, its radio transmission distance will be limited. In order to solve the energy consumption and distance problems during transmission, if the sensors are too far away from the base station, the WSNs need to organize a large number of distributed nodes to form a multi-hop wireless network. In WSNS, the sensor uses multiple-hop to establish network routing. The data is transmitted back to the base station through the shortest path composed of multiple sensors [4]. In addition, in a WSN since the wireless sensor is powered by a battery, an energy efficient and reliable load balancing is relatively important. There are many approaches to reduce energy consumption in WSNs [5, 10]. Such as load balancing among wireless sensor nodes, path selection when transmitting data, scheduling problems, sleep mechanism when there is no sensitive data, correctness of transmitting data, and so on $[11,34,35]$.

Generally, devices $\mathrm{Ci}$ belongs to the Wireless Sensor Networks (WSNs) have the capacity to organize them- selves to form an operational network without human intervention specifically in remote areas. These networks consist sensor nodes $C i$, base station or sink node(s) $S j$, actuator (where needed) and servers etc. These devices $C i$ are either deployed manually (known as engineered deployment) or randomly (usually through a helicopter or other means specifically in hard areas where manual deployment is not possible) in vicinity of the underlying phenomenon needed to be observed periodically or when a particular event occurs [1]. Due to the overwhelming characteristics of these networks, WSNs are used in different application areas such as military, smartbuildings, Medicine, agriculture, etc. to assist human beings in various real life-activities or automate it completely where applicable. Every member device $C i$, i.e., sensor node, prob the environment periodically or when an event is triggered and shares the collected data with the cluster head $(\mathrm{CH})$ or base station(s) or server(s) through a reliable communication mechanism that is either single-hop (in case of hierarchical WSNs) or multi hop (flat WSNs) [2]. In case of hierarchical networking infrastructures, wireless communication among various devices $C i$ is very simple as, usually, every device $C i$ is deployed within wireless communication range of the concerned base station module $S j$ [3]. Alternatively, in later case, each device $\mathrm{Ci}$ has to use path or rout information, i.e., load balancing or routing table, for the reliable transmission of data and it is due to the fact that majority of these devices are deployed far away from the concern base station and are unable to communicate directly [4,33]. Multi-hop communication is an ideal mechanism to resolve challenge of limited wireless communication that is closed related to the resource limited member nodes of operational WSNs. In this regard, various load balancing and routing schemes have been presented in literature to enhance the operational capabilities of these devices $C i$ specifically in terms of communication range, networks lifetime and end-to- end delay. 
Shortest path with minimum cost path enabled routing schemes are considered among the most reliable communication techniques. Specifically for those systems that consider timely delivery of data has more priority than connectivity of networks like intruder or malicious detection. In these schemes, shortest path are identified and every member node $C_{i} W S N s$ is force to transmit data only through those path(s). One of the common problems associated with shortest path enabled schemes shortest lifespan of device $C_{i}$ reside on the these shortest path which become the worst if multiple source nodes or devices share this shortest path for the transmission of packets [5], [6]. Hence, for the networks with limited resources, a uniform load balancing or traffic distribution strategy is always a challenging task specifically for the research community to resolve the aforementioned issue particularly with available resources. A well-known methodology that is multiple-path enabled load balancing schemes, which are primarily based on residual energy $E_{r}$ of neighboring devices $C_{i}$, were presented to distribute net- work traffic uniformly across different communication paths [7][10]. In this scheme, sensor nodes can send data to the neighbors whose hop-count values are greater than the sender node. However, end-to-end delay and packets delivery ratio are among the common problems associated with these schemes as maximum packets are transmitted via longest paths available in the operational WSNs. Likewise, criticality aware load balancing schemes was proposed in literature, which has resolved some of the afore mentioned issues associated with multiple path enabled schemes such as net- works lifetime, average packet delivery ratio and throughput [11], [12]. However, a tightly coupled issue associated with these approaches is packet loss ratio which will increase if neighbor node with minimum reliability factor is selected as a relay node. Therefore, an efficient and reliable mechanism for energy load balancing and communication is needed to be developed to address the aforementioned issues in WSNs without any change in the infrastructure of wireless technologies.

Moreover, the research community suggests various techniques to resolve the load balancing issue of WSNs and prolong the network lifetime. Although, these techniques are efficient at some stage to manage the load balancing issue, but they have some side effects on the network performance such as high communication cost, end-to-end delay, packet lost ratio, throughput, and individual sensor devices lifespan, etc. Therefore, our proposed scheme works based on (1) utilizing the maximum of the shortest path(s); (2) conducting a smart load balancing across multiple paths (particularly those with maximum residual energy, minimum round trip time (RTT) and minimum average packet loss ratio); (3) minimum load on nodes having low residual energy $\mathrm{Er}$ and lowest round trip time (RTT) RTTi ; and (4) maximum load on paths with least hop count (Hc value), maximum Er and minimum $\mathrm{Nv}$ value; which is largely not considered in many published models, in order to improve the performance and energy efficient for the networks that have limited resources in terms of average packet delivery ratio, networks lifetime.

The proposed model will investigate the potentials of the weighted ensemble based load-balancing scheme in resolving the aforementioned issues such as end- to-end delay; packets deliver ratio, and maximum lifetime. In this paper, a weightedensemble based load-balancing scheme is presented for WSNs to address the aforementioned issues. In the proposed scheme, different weight-ages are assigned to various metrics of every member node such as residual energy $E_{r}$, Hop-count $H_{c}$, crucial $N_{v}$ (from net- works connectivity perspective) and First Hop Neighbor Round Trip Time $\left(F N R T T_{i}\right)$. Moreover, the proposed scheme adopts a sequential approach such that first packet is sent through the most optimal path that is computed using those metrics. Second packet is forwarded on the $2^{\text {nd }}$ optimal path available in the operational WSNs.

The remaining paper is organized as follows. In subsequent section, a brief literature review is presented. In Section 3, the proposed mechanism is described in detail whereas results discussion is presented in subsequent section i.e., Section 4. Finally, concluding remarks and future directives are provided.

\section{RELATED WORKS}

In WSNs, which have various constraints, management of load balancing and optimized energy consumption of sensor devices are considered a vital role in enhancing the prolong network lifespan with better end-to-end delay, packet delivery, throughput, and packet loss ratio. Based on that, there is need to develop an efficient communication infrastructure of sensor devices with uniform load balancing to maximize its performance with optimal resource utilization, specifically for WSNs that have limited resources. To address this issue, many schemes have been proposed in the literature. Thus, in this section, the existing techniques of WSNs load balancing are comprehensively overviewed with their merits and demerits.

In the literature, there is an important question, How to develop an efficient load balancing scheme with minimal resources and maximum results for WSNs or IOTs? Proposing a new scheme based on a uniform load or traffic distribution approach over multi-path and multi-hop communication infrastructure to share the collected data of sensor devices in the network is a powerful solution for this issue in networks with limited resources like IOTs and WSNs. In this manner, we used both optimal-path identification scheme and gradient enabled scheme in the directed diffusion method, where server module or base station $S j$ chooses the optimal operational path; in this case, it will impose on the ordinary or member nodes $\mathrm{Ci}$ to utilize this path as a continuous communication link between sensor nodes. This path is used by various devices interested in transmission of data until one or more of these devices consume their on-board power completely or becomes not operational due to other reasons [13], [14]. Generally, this scheme relies on a single communication path, consequently, the lifetime of both WSNs and ordinary devices $C_{i}$ is very short, that is similar to the shortest path-enabled scheme where packets are forward on this path as long as all nodes reside on it are operational. Likewise, a shortest pathenabled approach was presented to resolve coverage area problem in mobile relay node(s) [15]. A common problem associated with these schemes is the selection and reinforcement of another optimal path that is needed in scenarios where existing path is no longer available or not 
reliable for further communication. This process is energy starving, time-consuming and costly for the resource-limited networks specifically WSNs and IoTs. A greedy approach enabled algorithm was used to develop a uniform load balancing to spread the traffic uniformly and enhances overall lifetime of the networks [16]. In the same way, Zheng et al., $[17,18]$, proposed a new scheme by computing the transmission capabilities for every sensing device $\mathrm{Ci}$ by their neighboring devices based on processing cost, hop count Hc, residual energy $\mathrm{Er}$ and round trip time (RTTi). At the beginning, to ensure reliable wireless communication, source device assigns a neighbor device $\mathrm{Ci}$ with lowest $\mathrm{Hc}$ value over others having highest $\mathrm{Hc}$ values. In addition, the scheme proposes that every device $\mathrm{Ci}$ should find at least 4 shortest and reliable paths, and forward maximum number of packets on the most two reliable and optimal routes. Therefore, unlike previous shortest path schemes, devices $\mathrm{Ci}$ available in these paths do not deplete their on-board battery more rapidly than other schemes through assigning weight-age factors accurately do. Software-Defined Wireless Sensor Network (SDWSN) architecture for load balancing of IoT was proposed by Cui et al. [19]. The proposed technique uses a centralized Software Defined Network (SDN) with flexible traffic management to minimize the energy consumption of participating IoT devices in the network. Moreover, for real traffic monitoring in the network, the authors also used the Open Flow protocol framework to verify the performance of the network. Qin et al. [20] proposed the software-defined network framework for load balancing in WSNs and IoT. Furthermore, they considered the emergency control system in the urban area to verify the feasibility of the proposed scheme.. Based on the results, they found that energy based traffic spreading approach have better performance as compared to other schemes.

Additionally, another study by Yousif et al., [21], they applied a weighted optimal path for loading balancing to solve the problem of uniform traffic distribution in wireless sensor networks. Another scheme known as aware load balancing scheme was proposed [11] to improve the network lifetime with ensuring the least possible percentage of end-to-end delay and packet delivery ratio. The performance of these schemes was exceptionally well, particularly in terms of networks lifetime enhancement metric, but it has some disadvantages like longer path selection to send maximum packets with continuous rate consumes extra energy and this creates network overhead.

The literature of WSNs in terms of load balancing schemes contain various cluster-based schemes or hierarchical networking infrastructures that have been used in the recent past to address the load balancing issues for networks with limited resources such as end to end delay, packet lost ratio, throughput, and sensor devices lifespan, coverage area, etc. [9]. Most of the researchers used the LEACH protocol in their proposed models to address the issue of load balancing in WSNs. However, one of the most difficult challenges in this type of networks is the identification the optimal cluster head node $(\mathrm{CH})$, which is responsible to send the collected data from sensor devices to the concerned base station. Although, LEACH protocol plays significantly will in the formation of the cluster head nodes in the network, but it has some disadvantages like dynamic cluster head nodes selection with continuous rate consumes extra energy and creates network overhead. In the same way, Zhang et al. [22] proposed a new load-balancing scheme for homogeneous networks, where both cluster head nodes ( $\mathrm{CHs})$ and member nodes $\mathrm{Ci}$ have the same features in terms of processing power and communication. The work of this scheme is based on moving the sensor nodes from $\mathrm{CH}$ s that have a heavy load to $\mathrm{CH}$ s with lowest load. The movement of sensor node is based on its location. In other word, it depends on the distance of sensor nodes, from the nearest $\mathrm{CHs}$ with highest volume of residual energy Er. A load-balancing scheme based on weighted based traffic was developed to solve the networks lifetime issue that have limited resources. To solve this issue, this scheme perform by selecting the $\mathrm{CH}$ based on its residual energy and number of deployed nodes in its vicinity. A stochastic distribution based traffic distribution scheme was proposed by Liao et al. [23], which aimed to generate a new model based on a uniform energy consumption throughout the networks. In the same way, another schemes based on tree approach were proposed to solve the load balancing problem and to establish a reliable communication infrastructure [18]. However, the majority of these schemes are either application specific or overlay complex or expensive due to change in the existing communication mechanisms. Therefore, a reliable load balancing mechanism is needed to resolve this issue with available resources.

Theory of information enabled load balancing scheme was presented to distribute traffic uniformly through a proper scheduling of modulation and routing mechanisms [24]. Additionally, Preethiya et al. [25] have proposed mobile double cluster-head enabled particle swarm optimization approach to address uniform traffic distribution challenge in heterogeneous WSNs which is divided into four phases such as $\mathrm{CH}$ selection, scheduling of $\mathrm{CHs}$, mobility and handover predication of a specific $\mathrm{CH}$ in the operational WSNs environment. However, complexity and implementation in realistic environments are among common issues associated with this approach. Zarin et al. [26] have presented a central controller node-enabled approach to distribute traffic uniformly throughout the networks where radio signals are utilized to balance energy consumption of active nodes in an operational heterogeneous WSN. A common issue with this approach is that reliable communication among mobile nodes and base station is not guaranteed. Similarly, a centralized optimal task allocation scheme is proposed to spread packets uniformly among available paths in the operational networks [27]. To realize this, linear programming and distributed optimal task allocation algorithms were integrated and utilized to minimize energy consumption of sensor nodes.

\section{A. Problem Statement}

With notable differences of existing studies [11], [7], [25], many schemes have been proposed for the load-balancing problem in WSNs. These schemes have not taken into consideration some important parameters like minimum possible average end-to-end delay and latency or maximum packet delivery ratio in order to ensure a reliable and optimal load-balancing scheme with an ideal wireless communication. 
In addition, Existing load balancing schemes were either performed on $\mathrm{CH}$ level, particularly in cluster based infrastructures, or in multiple path based scheme usually through Er, as in flat networks, but a generalized load balancing methodology, that is applicable in different networking infrastructures and topologies, is not addressed yet. Therefore, the proposed work will be focused on the development of a reliable neighborhood-enabled load balancing and communication scheme and its implementation in real environment of the resource-limited networks. Furthermore, the proposed scheme should be suitable and implementable in various topological infrastructures of WSNs. Additionally; the proposed work should thoroughly investigate criticality or importance of device $C_{i}$ particularly from networks connectivity perspective. Criticality of various nodes in the operational WSNs is depicted in Fig. 1 where communication activity of sensor nodes A, B, C and D are primarily dependent on the availability of sensor node $\mathrm{H}$. Therefore, a reliable communication mechanism is needed to be developed which should bound each and every sensor node transmit packet to a non-critical nodes (if available). For example, sensor nodes E, G, F and I should be forced to avoid transmission of packets via sensor node $\mathrm{H}$ as alternative routes are available for these nodes in Fig. 1.

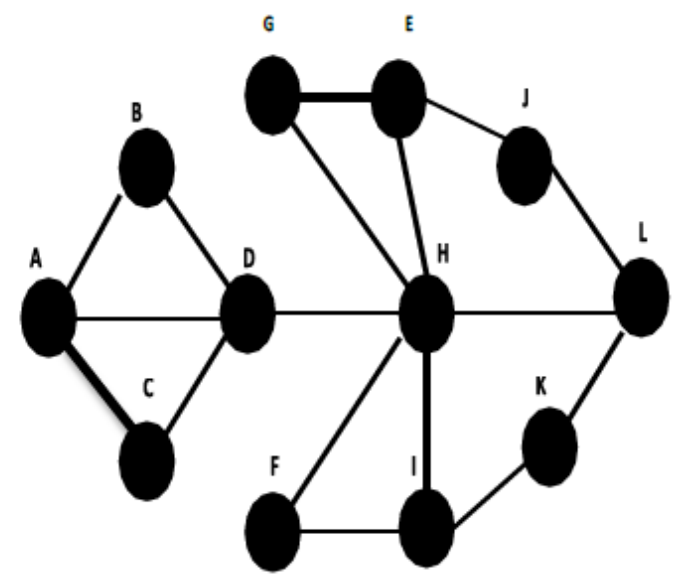

Fig. 1. Importance of Ordinary Sensor Nodes from Networks Connectivity Perspective.

\section{Computational Mechanism OF SENSOR NodeS CRITICALITY FACTOR (NV)}

Importance (criticality factor from the networks connectivity perspective) of a sensor node $C_{i} W S N s$ is a onestep process that is criticality of a member $C_{i}$ is computed only once i.e., when the networks deployment process is complete. Generally, the base station module is responsible to compute criticality factors of each and every member node $C_{i}$ and share it with these nodes. For this purpose, various concept of graph theory (preferably planner graph) are used to compute this factor. Higher value of a sensor node $C_{i}$ criticality factor $N_{v}$ is an indication of how important that node is from the WSNs connectivity perspective? For example in Fig. 1, criticality factor value $N_{v}$ of sensor node $\mathrm{H}$ is higher than other nodes as a certain portion of the underlined network (i.e., A, B, C and D) are completely dependent on its active- ness for the transmission of packets. Moreover, this factor play a vital role to distribute traffic in a biased but efficient manner (from residual energy $E_{r}$ perspective). Therefore, the proposed load balancing mechanism should be smart-enough to minimize traffic flow on paths or neighboring sensor nodes $C_{i}$, which have higher values of criticality factor than other nodes in the operational WSNs. A detailed description of the methodology that is used to calculate criticality of sensor nodes $C_{i} \in W S N s$ is given below.

\section{A. Calculation of Levels in WSNs}

The computation process of a sensor node $C_{i}$ criticality factor is initiated with the calculation of levels in WSNs preferably before and after removal of the concerned node $C_{i}$. For this purposed, a well-known technique called logical networks abridgment (LNA) is adopted which is used to generate loop-free description of the underlined WSNs [28]. Moreover, the concept of planner graph is utilized to develop a proper sketch of the deployed WSNs as shown in Fig. 2 where various level are identified such as level-1, level-2, etc. Usually, the deployed WSNs physical infrastructure is represented by Level-1, which is $1^{s} t$ level which is generated through the planner graph concept. An active sensor node $\mathrm{Ci}$ and communication link between two neighboring nodes in WSNs corresponds to a vertex and an edge in a graph respectively. A closed ring in the underlined graph should be a vertex in the next level as shown in Fig. 2 where an edge in the next level is subjected to neighborhood. A sensor node's levels calculation process is assumed to be completed iff a graph with no loop or cycle, a closed proximity of three or more nodes, is generated as represented in green at level-3 in Fig. 2. Level-2 graph is generated from level-1 where every vertex belongs to this level corresponds is created only if there is a cycle in level-1 graph and an edge is drawn iff there exist a pair of vertices belong to level-1 reside in closed proximity or neighbors. Moreover, these pair of vertices should belong to a similar bi-connected graph. In scenarios where these cycles do not belong to the same bi- connected graph then.

1) An edge in level-2 is drawn if and only if these cycles have a common vertex or node, which is known as cut- node in graph theory.

2) If these cycles do not have any common node(s), then edge should not be added.

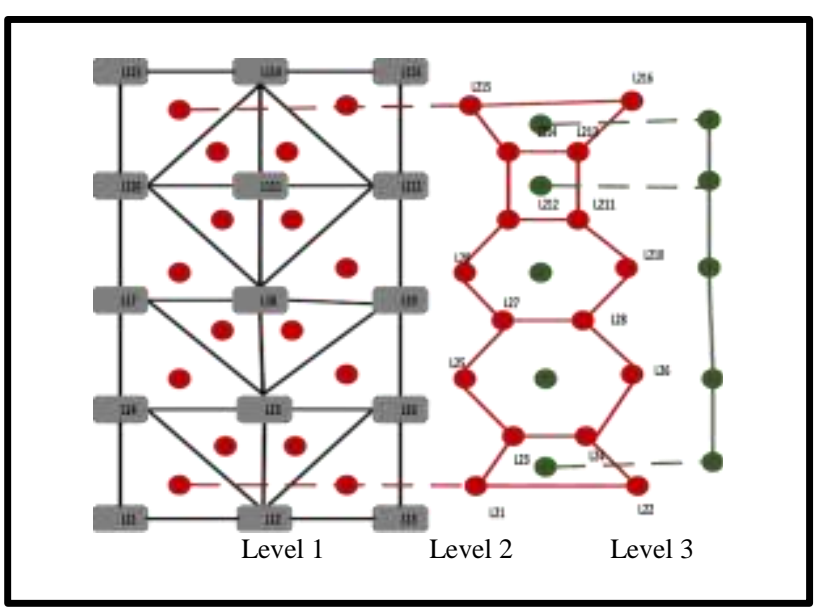

Fig. 2. Sensor Node Criticality Metric Computation Mechanism. 
The development process of Level-2 from Level-1 is identical to that of a dual graph where a vertice, which is surrounded by at-least three vertices (preferably to form a ring or cycle), is considered as a vertice in the next level exterior nodes are excluded and interior nodes are considered as shown in Fig. 2. Similarly, Level-3 vertices and edges are formed using this procedure. This process of levels computation is repeated until a loop free structure (a planner graph with no cycles) of the underlined graph is obtained as shown in green in Fig. 2.

After computing number of levels in the entire WSNs, the next phase is calculate these levels for each and every member node $\mathrm{Ci} \in W S N s$. For this purpose, a sensor node for which levels are to be computed is assumed to be removed (temporarily) from the WSNs. For example in Fig. 1, if levels are to be computed for sensor node say 'A', then this node is assumed as removed or non-member from this network which becomes as shown in Fig. 3. Then the above mentioned process for whole networks levels computation is repeated for this network. In this way, levels of whole networks and member nodes are computed.

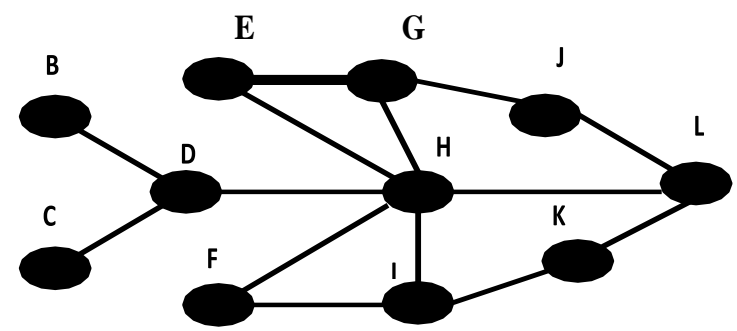

Fig. 3. Level Computation of an Individual Sensor Node.

\section{B. Criticality of Member Sensor Nodes: Calculation Methodology}

Criticality metric of a sensor node plays a vital role in prolonging the WSNs connectivity and, hence, enhancing their lifespan. Criticality metric of sensor nodes $C_{i}$ WSNs is computed using the following formula.

$\mathrm{v}_{\mathrm{n}}=\frac{\mathrm{N}_{\mathrm{B}}}{\mathrm{N}_{\mathrm{A}}} \times \frac{\mathrm{L}_{\mathrm{B}}+\mathrm{C}}{\mathrm{L}_{\mathrm{A}+\mathrm{C}}}$

Where $V_{n}$ is the criticality factor of $n^{\text {th }}$ sensor node in an operational WSN. $\mathrm{N}_{\mathrm{B}}$ and $\mathrm{N}_{\mathrm{A}}$ represents number of nodes in network before and after removing $\mathrm{n}^{\text {th }}$ node respectively. For example, there are twelve nodes in WSNs as shown in Fig. 1. Whereas eleven nodes remains after removing sensor node A from the underlined WSN as shown in Fig. 3. Likewise, $\mathrm{L}_{\mathrm{B}}$ and $\mathrm{L}_{\mathrm{A}}$ represent levels in WSN before and after removing that node respectively. For example in Fig. 4, both networks have same number of sensor nodes i.e., five. Networks-A is a flat network with single level i.e., each node has a single path of communication. However, in network-B, each sensor nodes has two different paths available for communication. Removal of a node, as described above in level calculation mechanism, from a deployed WSN is used to examine how networks connectivity is effected if that particular node consumes it onboard battery completely. For example, if we remove sensor node $\mathrm{C}$ from both networks then nodes belong to network-A will not able to communicate with each other whereas those belong to network-B are able to communicate, as alternative path is available as shown in Fig. 4. Once criticality metric of every sensor node $C_{i}$ is completed then the base station $S_{j}$ module shares these values with its member sensor nodes. Thus, each and every sensor node stores criticality values of its neighboring devices along with other information.

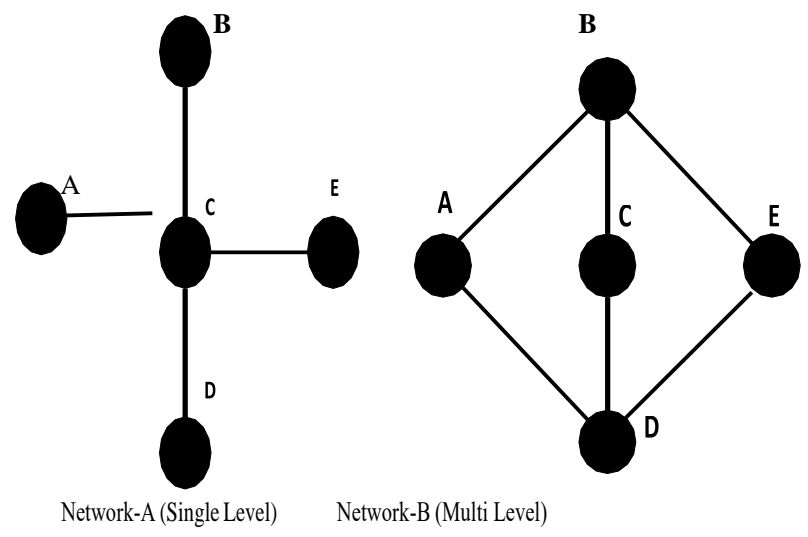

Fig. 4. Effects of Levels on the Networks Connectivity.

\section{PROPOSED NeIGHBorhood-ENABLED LOAD BALANCING SCHEME FOR WSNS}

A neighborhood-enabled load-balancing scheme, which is specifically designed for the wireless sensor networks, is presented to distribute traffic uniformly across all available paths in an operational network where every sensor node is bounded to send packet via reliable and non-critical paths or nodes. To achieve this goal, various routing metrics related to the neighboring nodes are used such as hop-count $H_{c}$, residual energy $E_{r}$, Round Trip Time (RTT) and sensor node criticality $N_{v}$.

\section{A. Hop-Count Discover Phase}

Hop-count information plays a vital role in resolving the ping-pong (multiple transmission of same message again and again) between neighboring node in operational WSNs environment. For example, a sensor node will discard a message received from neighboring device whose hop-count value is less than its own. In this phase, a control packet, $M s g_{h d}$, is generated and broad casted by the base station module $S_{j}$, with value of $H_{c}=0$ as base station is ultimate destination of all packets, which is received by its neighboring nodes. These nodes update their $H_{c}$ according, that is $H_{c}=1$, and broad-cast an updated version of that message $M s g_{h d}$, i.e., with value of $H_{c}=1$, after its back-off time expires. Back-off timer is used to minimize the collision probability of these messages.

$T_{b}\left(C_{i}\right)=\operatorname{rand}(0-1000)+\min \left(H_{c}\left(C_{i}\right), \delta\right)$

The idea of adding an $H c$ value or $\delta$ with the generated random number is to further minimize (or avoid if possible) the packets collision probability of neighboring nodes as usually, these nodes have different $H c$ values. However, in scenarios where back-off timer $\mathrm{Tb}$ of two or more neighboring nodes are similar, then these nodes $\mathrm{Ci}$ should re-compute their back-off timer $\mathrm{Tb}$ accordingly. Moreover, parameter $\delta$ is an application dependent value whose values are different for different topological infrastructures such as in flat networks its value is in between 5 and 15 whereas between 2 and 5 for the hierarchical WSNs. This process is repeated by each and every 
member sensor node $\mathrm{Ci}$ WSNs until $H c$ value is collected by every member device in the WSNs.

Moreover, every sensor node $C_{i}$ keeps a record of it neighboring nodes along with their hop-count values. For this purpose, a simple table is maintained where this information is recorded from time to time. Separate session is not needed for this purposed rather it is performed as sub part of hop- count discovery phase. For example, when a sensor node receive message $M s g_{h d}$ that contains information about it source such as hop-count and MAC-ID. Every sensor node records this information in a table, which is used to identify optimal and reliable neighboring nodes for the transmission of packets.

\section{B. Finding Optimal Neighboring Nodes}

In the proposed setup, an optimal neighboring node $C_{i}$ $W S N s$ is defined as a sensor node with maximum residual energy $E_{r}$ and minimum possible (hop-count $H_{c}$, criticality $N_{v}$ $\&$ Round Trip Time (RTT) values. In next phase, these nodes find two optimal neighbors or paths, preferably with minimum hop-count $H_{c}$, lowest $R T T_{i}$ value, minimum $N_{v}$ value and maximum residual energy $E_{r}$. These paths are used in the biased way (such as forwarding maximum traffic on most optimal path i.e., $70 \%$ whereas $30 \%$ on other path) to increase average packet delivery ratio and decrease the overall packet lost ratio and end-to-end delay of packets in an operational WSN. It is to be noted that packet delivery ratio is improve if majority of the packets are sent via most optimal path. Moreover, it is quite likely that packet loss ratio is minimize due to forwarding packets over a most reliable path or neighbor.

In order to identify optimal neighboring node(s), different weight-ages are assigned to the hop-count $H_{c}$, residual energy $E_{r}$, RTT and Criticality $N_{v}$ values. Initially, maximum weightage is assigned to the hop-count and criticality $N_{v}$ metrics of various neighboring nodes to forward maximum packets or traffic through the shortest and less critical path. Moreover, specifically at this stage, residual energy $E_{r}$ of all neighboring nodes are similar, therefore, it will not affect the optimal neighbor's' selection process irrespective of higher or lower weight-age value assigned to it. Optimal neighboring node selection process is subjected to the following (3):

$O p t_{\text {Neighbor }}=\operatorname{Min}\left(W_{1} \times H_{c}+W_{2} \times N_{v}+W_{3} \times E_{r}+W_{4} \times R T T_{i}\right)$

Whereas $W 1, W 2, W 3, W 4$ are the weight-age factor assigned to the aforementioned metrics. In proposed setup, initially these values were assigned i.e., $W 1=30 \%, W 2=$ $40 \%, W 3=10 \%$ and $W 4=20 \% . N_{v}$ value represents the criticality of a member node from the resource limited networks connectivity factor i.e., how important is a particular node $C_{i}$ as far as WSN's long term connectivity is concerned. As described above that a higher value of criticality $N_{v}$ associated with a node is an indication that a certain portion of the underlined WSN either partially or completely rely on this node i.e., if it becomes non-active (by consuming its on- board battery) then the portion of WSN will be disconnected or will not be able to communicate properly with the base station module $S_{j}$. Moreover, $O p t_{\text {Neighbor }}$ is the node $C_{i}$ with (probably) a shortest path to the destination module i.e., base station.
Initially, packets generated by various source nodes $C_{i}$ are distributed uniformly between two optimal neighboring nodes i.e., $50 \%$ packets forwarded through $O p t_{\text {Neighbor } 1}$ and $O p t_{\text {Neighbor } 2}$ respectively. However, the proposed scheme should avoid (if possible) critical nodes even though these are the most optimal neighbors. A neighboring sensor node $C_{i}$ is assumed to be critical iff

1) Certain portion(s) of the underlined WSNs are either partially or completely dependent on that neighbor for the transmission of packets.

2) It acts as the shared optimal path among various nodes in the WSNs.

3) It has consumed approximately $80 \%$ of its on-board battery.

Moreover, if two or more neighboring nodes have a similar optimal value, then the selection process is random. Forwarding packets on most reliable path, a path with zero critical node, results in enhancing lifespan of the underlined constraint oriented networks. Additionally, the WSNs become connected for maximum possible duration without changing in the technological infrastructure.

The weight-age ratio presented in equation. 3 is maintained by every sensor node $C_{i}$ until one or more of its neighboring nodes consume $75 \%$ of their on-board batteries. $75 \%$ threshold value is selected due to the fact that this node will be active for maximum possible duration. This node is now dedicated to forward data or packets of those nodes that have no other path(s) or neighbor(s) toward the base station module $S_{j}$. As soon as a sensor node $C_{i}$ broadcast a message which indicates it critical condition i.e., that it has consumed $75 \%$ of the available power, then neighboring node(s) readjust their packets forwarding schedule or methodology accordingly. In this scenario, criteria for finding optimal neighboring nodes is revised in equation 3 by adjusting their weight-age factors such as residual energy $E_{r}$ is assigned highest weight-age followed by nodes criticality value $N_{v}$. These weight-ages are adjusted as follows. $W 1=10 \%, W 2$ $=30 \%, W 3=40 \%$ and $W 3=20 \%$. Now, maximum packets are routed through those neighbors, which have maximum possible residual energy $E_{r}$ and minimum criticality value $N_{v}$. This re-adjustment policy not only resolves end- to-end delay issue that is linked to the WSNs but at the same time improve their lifespan considerably.

\section{Neighborhood-Enabled Load Balancing Algorithm}

Generally, criticality of a sensor node $C_{i}$ is high if it has maximum number of neighboring nodes particularly those nodes that have the capacity to communicate directly with it. Thus, it is quite likely that criticality of node $C_{i} W S N s$ is strongly correlated to its congested neighborhood. A node with maximum neighbors has a higher probability to forward a slightly higher ratio of packets than other nodes, i.e., node(s) with minimum neighboring nodes. Therefore, critical node(s) consume their on-board battery more rapidly than other nodes due to the heavy load. Therefore, a trade-off metric is implemented to relieve these nodes either partially or completely (if possible) with an acceptable performance degradation ratio in terms of transmission delay, energy 
consumption etc. The proposed load-balancing algorithm, which is presented below as Algorithm. 1, bounds every sensing device to thoroughly evaluate its neighborhood in terms of criticality, reliability, power capacity and distance before initiating a communication session.

\section{Computational Complexity of the Proposed Load Balancing Scheme}

Computational complexity is an evaluating metric that is used to describe the potentials and requirements of an underlined algorithm, in terms of time and space, to resolve a specific problem. Complexity of an algorithm has a direct corelation to its time and space requirements.

\section{RESUlTS OF SIMULATION AND DISCUSSION}

In this section, a detailed analysis of the simulation results is presented to evaluate the performance of the proposed neighborhood-enabled load balancing algorithm in terms of end-to-end delay, packet delivery-ratio, uniform load balancing (where possible), energy efficiency and residual energy (Er). The proposed LBS is compared with the field proven techniques i.e., the shortest path algorithm, energy based traffic spreading approaches [8], [29], opportunistic routing [30], and vulnerability aware routing [11], [12]. These algorithms were implemented in OMNET++ [31], an open source simulation environment that is specifically designed for the resource-limited networks. It is to be noted that similar processing and communication power, on-board batteries, a single gateway module were used both for the proposed and existing schemes. Moreover, these schemes were tested using different topological structures such as graph based topology, tree based, random-top, and random- center. Additionally, channels or paths delay factors; both path loss ratio and interference are assumed to be constant for the WSNs. A WSN whose nodes (preferably $\mathrm{n}$ in this case) are distributed randomly in a deployment area of $500 \mathrm{~m} * 500 \mathrm{~m}$ as shown in Table I. Moreover, we have assumed that a sensor node either communicates directly with the base station module iff it resides in closed proximity of the base station or through various relay nodes using multi-hop communication. Additionally, for simplicity the capacities of on-board battery and transceivers are similar to the wasp- mote agriculture proboard capacity [32] i.e., $52000 \mathrm{mAh}$ and 400 meters respectively. To streamline the proposed neighborhoodenabled load balancing scheme applicability in the realistic environments of WSNs, well-known ratios are used both for packets transmission and reception i.e., $P_{T X}=91.4 \mathrm{~mW}$ and $P_{R x}=59.1 \mathrm{~mW}$ respectively. More- over, initial hop-count of ordinary nodes $C_{i}$ is equal to ( ). In the proposed scheme, every node $C_{i}$ is needed to inform its neighbor's iff its residual energy $E_{r}$ is less than the defined threshold value i.e., $85 \%$ in this case. In proposed setup, the distance between various nodes $C_{i}$ is kept around 400 meter. Moreover, criticality factor of WSNs and individual sensor nodes is computed only once i.e., precisely after the deployment process. As described above, this process is performed by the gateway or base station module.

The proposed scheme were thoroughly examined against various fields proven schemes (preferably load balancing and routing) in term of residual energy $E_{r}$, lifetime, end-to-end delay, average packet delivery ratio and sent or forwarded packets.

\section{A. Uniform Energy Consumption}

The proposed scheme was designed and developed not only to distribute traffic uniformly across the networks (WSNs), specifically via reliable communication path(s), but at the same time generated traffic is distributed in such a way that each and every node consume approximately similar power i.e., their residual energy is approximately similar (preferably of neighboring nodes). The proposed scheme performance is far better than existing schemes as shown in Fig. 5.

TABLE I. HOMOGENEOUS WSNS SIMULATION PARAMETERS SETUP

\begin{tabular}{|l|l|}
\hline Parameters & Values \\
\hline WSN Deployment Area & $500 \mathrm{~m} * 500 \mathrm{~m}$ \\
\hline Sensor Node & $40,200,300,500$ \\
\hline Base Station & One \\
\hline Initial Energy $\left(\mathrm{E}_{s}\right)$ & $52000 \mathrm{mAh}$ \\
\hline Residual Energy $\left(\mathrm{E}_{r}\right)$ & $\mathrm{E}_{s}$ - $_{\text {consumed }}$ \\
\hline Packet Transmission Power Consumption $\left(P_{T X}\right)$ & $91.4 \mathrm{~mW}$ \\
\hline Channel Delay $\left(C h_{d e l a y}\right)$ & $10 \mathrm{millisecond}$ \\
\hline Packet Receiving Power Consumption $\left(P_{R x}\right)$ & $59.1 \mathrm{~mW}$ \\
\hline Idle Mode Power Consumption & $1.27 \mathrm{~mW}$ \\
\hline Sleep Mode Power Consumption & $15.4 \mu \mathrm{W}$ \\
\hline Transceiver Energy $\left(T_{i}\right)$ & $1 \mathrm{~mW}$ \\
\hline Transmission Range $\left(T_{r}\right)$ & $200 \mathrm{~m}$ \\
\hline Receiving Power Threshold $\left(R T S_{n}\right)$ & 1024 bits \\
\hline Packet Size $\left(P_{\text {size }}\right)$ & $127 \mathrm{bytes}$ \\
\hline Hop Count $\left(H_{c}\right)$ of Base Station & 0 \\
\hline Initial Hop Count $\left(H_{c}\right)$ of Sensor Nodes & \\
\hline Initial Criticality Value $\left(V_{n}\right)$ of Sensor Nodes & 0 \\
\hline Maximum Distance between Nodes & $100 \mathrm{~m}$ \\
\hline Sampling Rate of sensor nodes & $30 \mathrm{Seconds}$ \\
\hline
\end{tabular}

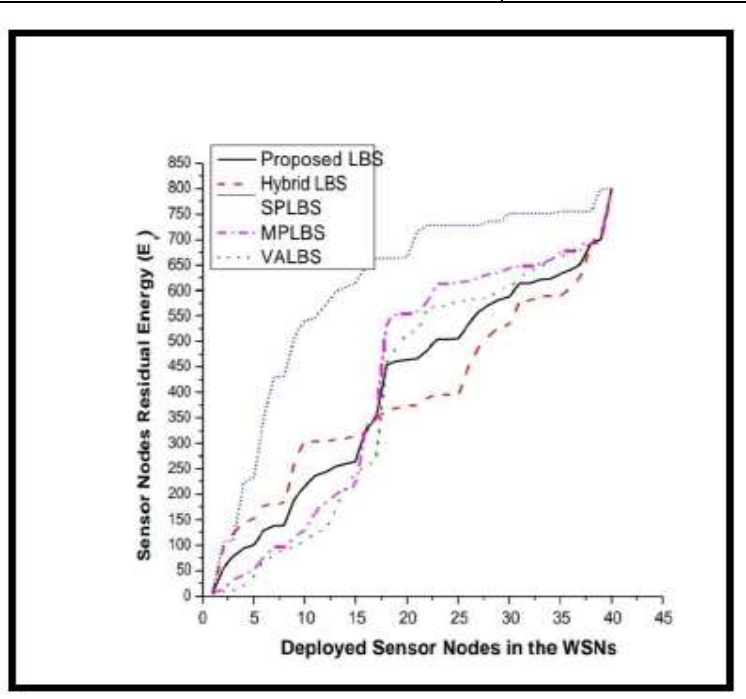

Fig. 5. Residual Energy of Sensor Nodes belong to the Operational WSNs. 
Algorithm 1 Proposed Neighborhood-enabled Load Balancing Algorithm

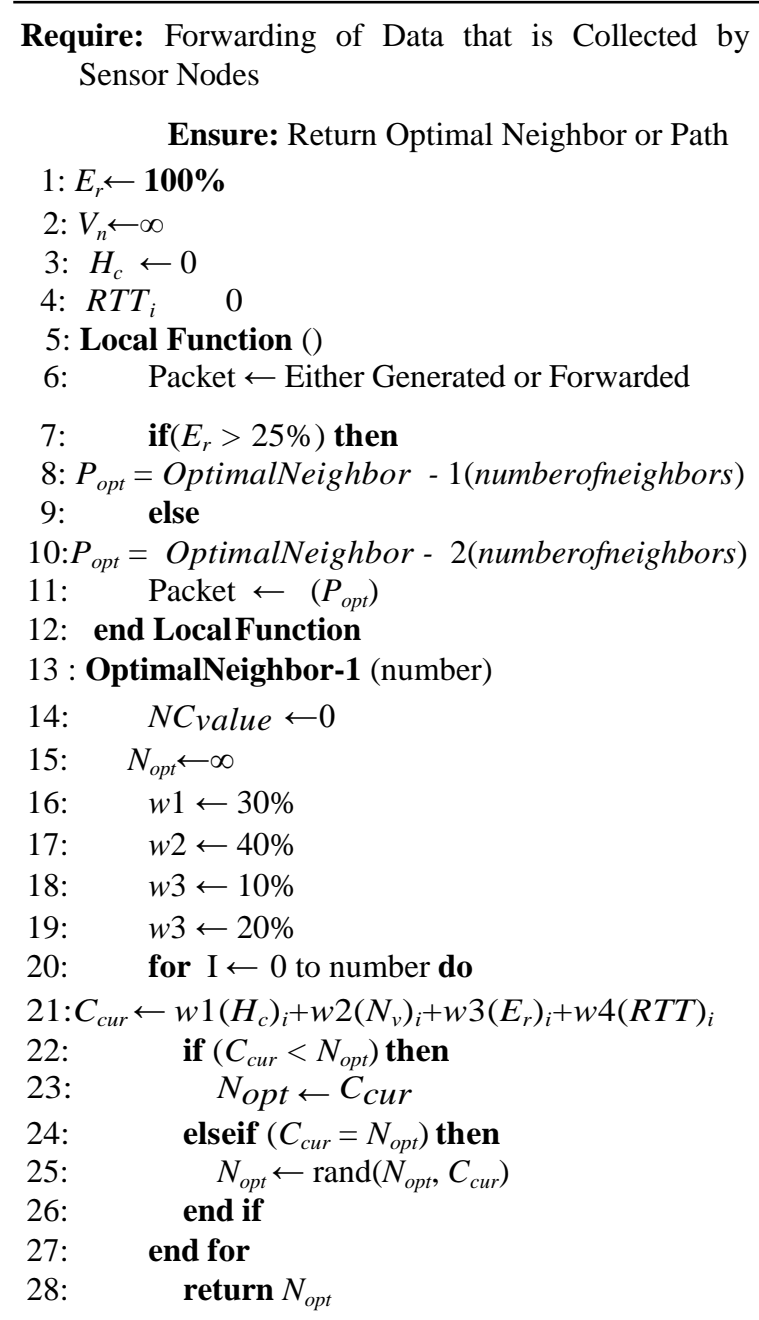

\section{9: END NEIGHBORDISCOVERY-1}

\section{0: OptimalNeighbor-2 (number)}

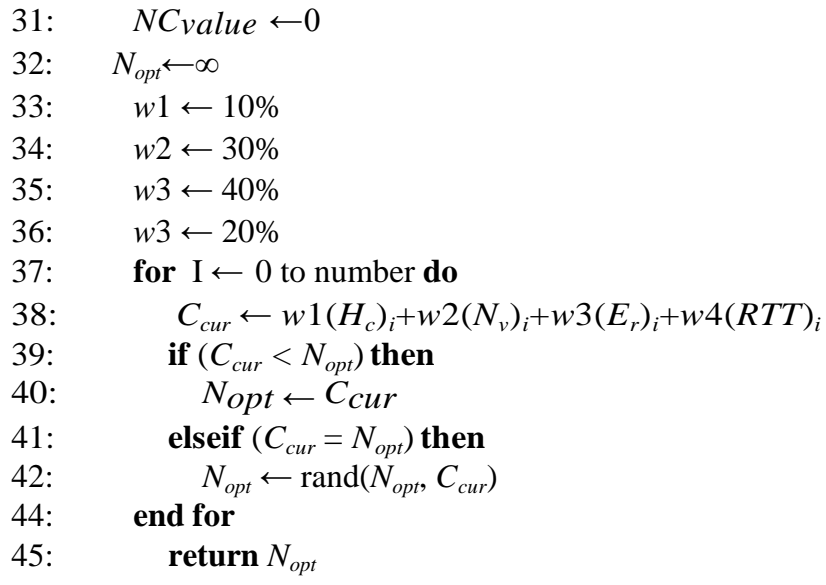

\section{6: END NEIGHBORDISCOVERY-2}

47: end

\section{B. WSNs Lifetime}

A prolonged lifetime of the resource-limited networks, WSNs and IoTs, is a challenging issue up to date. In literature, various mechanisms have been proposed to address this issue. It is evident from Fig. 6 that the proposed scheme is ideal solution for WSNs as it outperform the existing schemes. The WSNs lifetime is enhanced by the proposed scheme that is subjected to strong emphasis of the proposed neighborhoodenabled load balancing scheme on the avoidance (if possible and applicable only) of critical nodes during the communication activity. Moreover, as WSNs lifetime is subjected to the lifespan of individual nodes. Therefore, a brief analysis of an individual node lifetime is presented in Fig. 7. Lifetime of both individual node and whole WSNs is inversely proportional to the sampling rate i.e., a highest sampling interval leads to the minimum lifespan of WSNs.

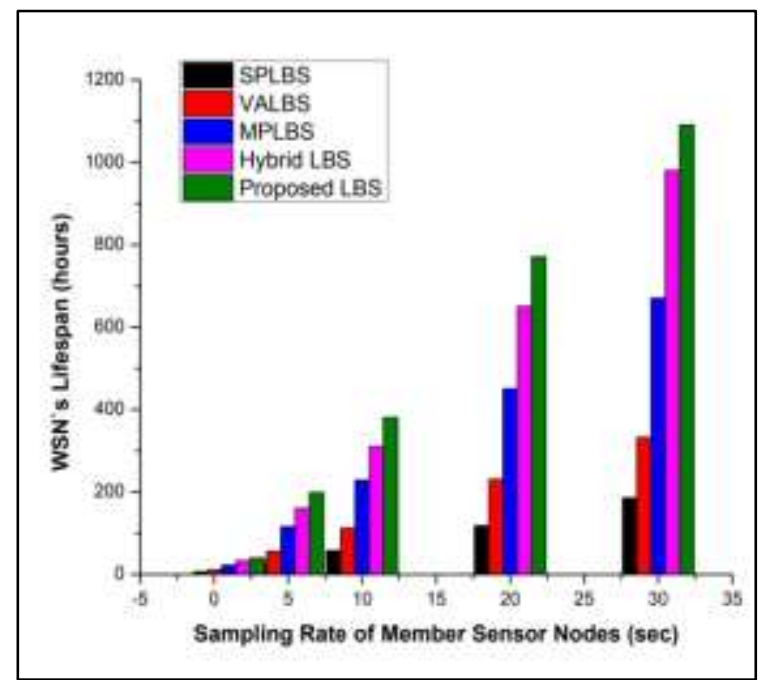

Fig. 6. Lifetime Comparison of the Proposed Neighborhood-Enaod-Enabled LBS and Field Proven Algorithms.

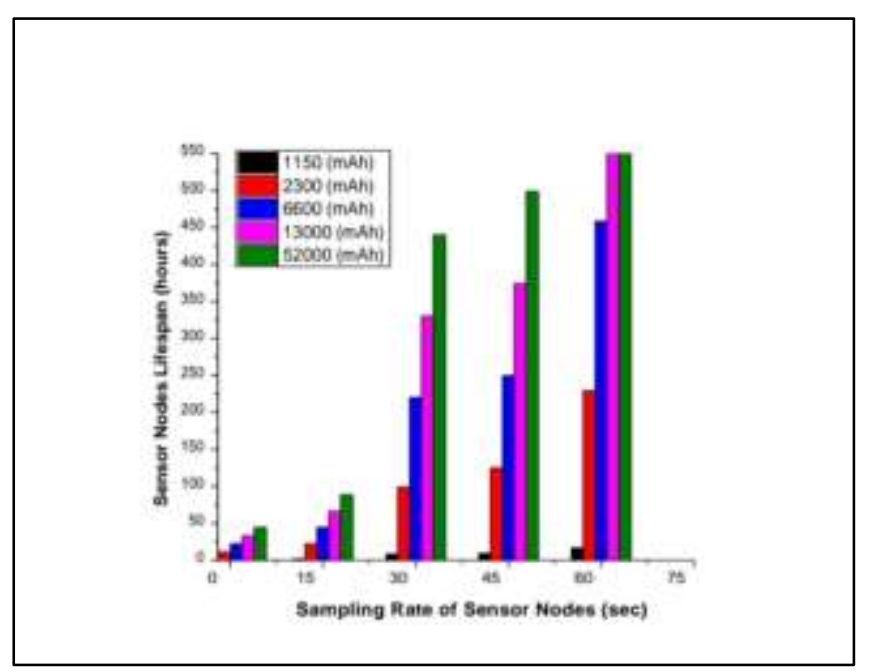

Fig. 7. Sensor Nodes Capacities on different Standard of Waspmote Onboard Batteries i.e., 1150, 2300, 6000, 13000, and $52000 \mathrm{mAh}$. 


\section{End-To-End Delay}

End-to-End delay is a basic measure to evaluate the performance of a load-balancing scheme specifically in the resource limited networks environments. A comparison of the pro- posed neighborhood-enabled LBS and existing algorithms in terms of average end-to-end delay performance metric is presented in Fig. 8, which shows that the proposed scheme has the lowest possible end-to-end delay metric than existing schemes specifically on various WSNs densities that number of member nodes. The proposed LBS achieves this milestone by selecting an appropriate path for an individual packet by considering different parameters of the neighboring nodes such as criticality factor, residual energy, and hop count etc. Additionally, Fig. 8 shows that end-to-end delay factor is directly proportional to the networks size, channel delay, queuing delay, transmission delay, receiving delay, and the location of source and destination devices.

\section{Average Packet Delivery Ratio}

Average packet delivery ratio is another important performance metric that is utilized to evaluate various load balancing schemes in the realistic environment of WSNs. Fig. 9 shows the comparison of average packet delivery ratio of the proposed neighborhood-enabled LBS with the existing schemes, which shows that the proposed scheme has maximum possible average packet delivery ratio. Moreover, these results were verified on various WSNs infrastructures such as different number of nodes $C_{i}$ and topologies. It is to be noted that the proposed neighborhood-enabled LBS algorithm achieves the highest ratio by utilizing a smart neighbor (preferably relay node) selection criteria and thus reducing the packet loss ratio.

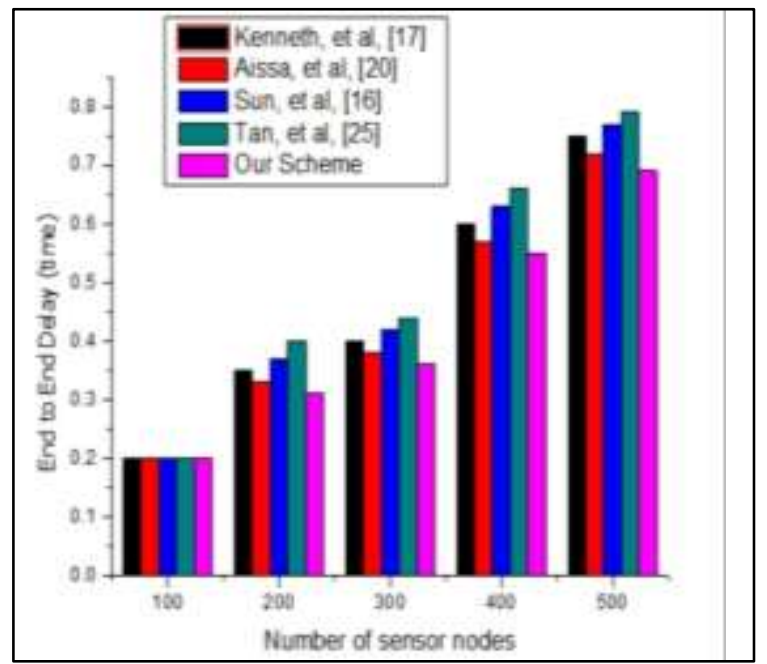

Fig. 8. End-to-End Delay Analysis Statistics of the Proposed and Existing Load Balancing Schemes.

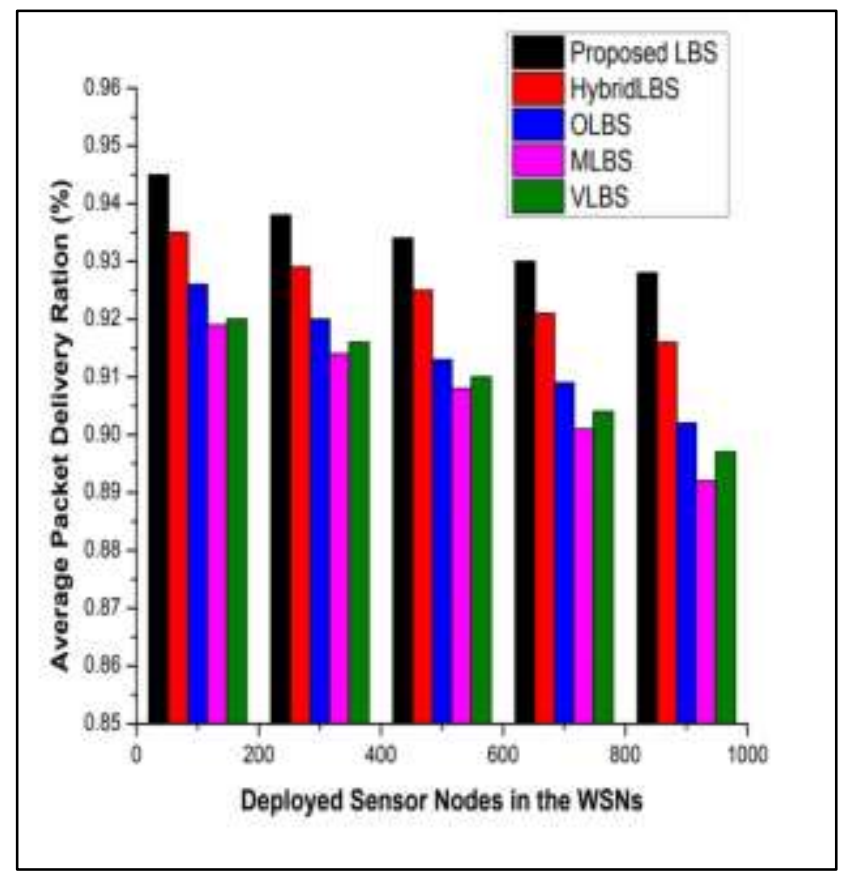

Fig. 9. Average Packet Delivery Ratio Analysis Statistics of the Proposed and Existing Load Balancing Schemes.

\section{E. Throughput Analysis}

An alternative evaluation metric is the throughput that is defined as total number of successfully delivered packets to the base station module in the WSNs. A load-balancing algorithm is considered as efficient and reliable iff it has achieved maximum throughput with available resources. The proposed scheme performs better than existing scheme in different realistic scenarios of the WSNs as shown in Fig. 10. The proposed neighborhood-enabled LBS has achieved the milestone of maximum possible throughput by bounding every node $C_{i}$ to send packet(s) via most reliable and preferably shortest path(s) or neighbors.

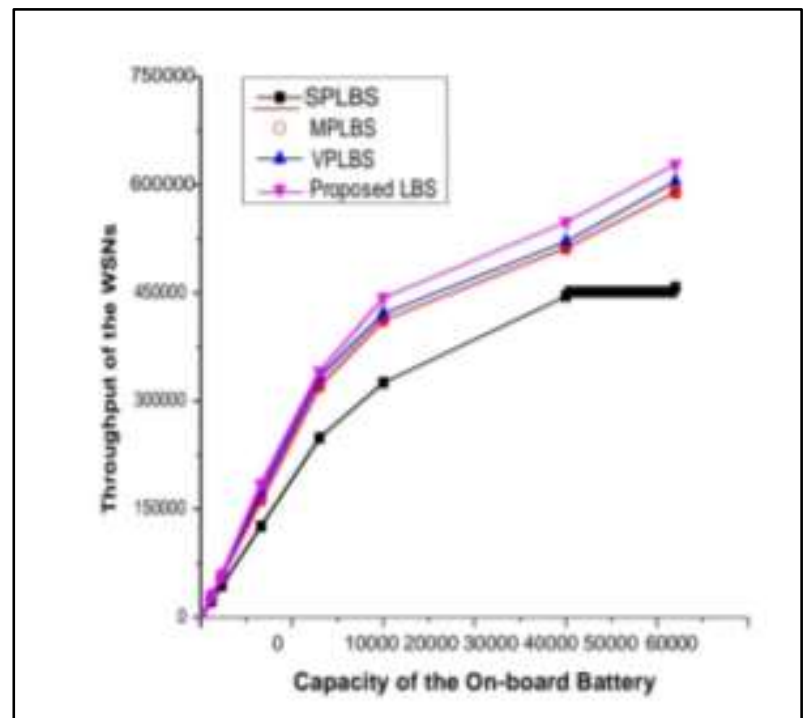

Fig. 10. Throughput Analysis Statistics of theProposed and Existing Load Balancing Schemes. 


\section{CONCLUSIONS}

In this paper, a neighborhood-enabled load-balancing scheme was presented for wireless sensor networks to resolve various issues associated with the existing schemes. Moreover, the proposed scheme did not compromise on other performance metrics such as end-to-end delay, lifetime, average packet delivery ratio and throughput of the concerned WSNs. The proposed neighborhood-enabled load balancing scheme assigns different weight-ages to various metrics or parameter of every neighboring node such as residual energy $E_{r}$, Hopcount $H_{c}$, crucial $N_{v}$ (from networks connectivity perspective) and first hop neighbor round trip time $\left(F N R T T_{i}\right)$. Moreover, the proposed load-balancing scheme adopts a sequential approach such that first packet is sent through the most optimal path that is computed using those metrics and next packet is forwarded on the $2^{\text {nd }}$ optimal path available in the operational WSNs. Simulation results have verified the exceptional performance of the proposed load balancing scheme against the existing LBS approaches particularly in terms of end-to-end delay, average packet delivery ratio, lifetime (both individual and whole WSNs) and throughput.

\section{REFERENCES}

[1] X. Liu, R. Zhu, A. Anjum, J. Wang, H. Zhang, and M. Ma, "Intelligent data fusion algorithm based on hybrid delay-aware adaptive clustering in wireless sensor networks," Future Generation Computer Systems, vol. 104, pp. 1-14, 2020.

[2] Almaiah MA, Dawahdeh Z, Almomani O, Alsaaidah A, Al-khasawneh A, Khawatreh S. A new hybrid text encryption approach over mobile ad hoc network. International Journal of Electrical and Computer Engineering (IJECE). 2020 Dec;10(6):6461-71.

[3] F. Wu, X. Li, L. Xu, P. Vijayakumar, and N. Kumar, "A novel threefactor authentication protocol for wireless sensor networks with iot notion," IEEE Systems Journal, 2020.

[4] M. Sajwan, D. Gosain, and A. K. Sharma, "Camp: cluster aided multipath routing protocol for wireless sensor networks," Wireless Networks, vol. 25, no. 5, pp. 2603-2620, 2019.

[5] X. Liu, T. Qiu, and T. Wang, "Load-balanced data dissemination for wireless sensor networks: A nature-inspired approach," IEEE Internet of Things Journal, vol. 6, no. 6, pp. 9256-9265, 2019.

[6] L. Cheng, J. Niu, C. Luo, L. Shu, L. Kong, Z. Zhao, and Y. Gu, "Towards minimum-delay and energy-efficient flooding in low-dutycycle wireless sensor networks," Computer Networks, vol. 134, pp. 6677, 2018.

[7] S. K. Singh, M. Singh, D. K. Singh et al., "Routing protocols in wireless sensor networks-a survey," International Journal of Computer Science \& Engineering Survey (IJCSES), vol. 1, no. 2, pp. 63-83, 2010.

[8] A. Laouid, A. Dahmani, A. Bounceur, R. Euler, F. Lalem, and A. Tari, "A distributed multi-path routing algorithm to balance energy consumption in wireless sensor networks," Ad Hoc Networks, vol. 64, pp. 53-64, 2017.

[9] H.-Y. Kim, "An energy-efficient load balancing scheme to extend lifetime in wireless sensor networks," Cluster computing, vol. 19, no. 1, pp. 279-283, 2016.

[10] A. R. M. Kamal and M. A. Hamid, "Supervisory routing control for dynamic load balancing in low data rate wireless sensor networks," Wireless Networks, vol. 23, no. 4, pp. 1085-1099, 2017.

[11] A. Sari and E. Caglar, "Load balancing algorithms and protocols to enhance quality of service and performance in data of wsn," in Security and Resilience in Intelligent Data-Centric Systems and Communication Networks. Elsevier, 2018, pp. 143-178.

[12] R. Khan, "An efficient load balancing and performance optimization scheme for constraint oriented networks," Simulation Modelling Practice and Theory, vol. 96, p. 101930, 2019.
[13] R. Khan, M. Zakarya, Z. Tan, M. Usman, M. A. Jan, and M. Khan, "Pfars: Enhancing throughput and lifetime of heterogeneous wsns through power- aware fusion, aggregation, and routing scheme," International Journal of Communication Systems, vol. 32, no. 18, p. e4144, 2019

[14] Y. Yuan, W. Liu, T. Wang, Q. Deng, A. Liu, and H. Song, “Compressive sensing-based clustering joint annular routing data gathering scheme for wireless sensor networks," IEEE Access, vol. 7, pp. 114 639-114 658, 2019.

[15] D. Cheng, Y. Song, Y. Mao, and X. Wang, "Lddp: A location-based directed diffusion routing protocol for smart home sensor network," in Systems and Informatics (ICSAI), 2014 2nd International Conference on. IEEE, 2014, pp. 510-514.

[16] C. N. Abhilash, S. H. Manjula, R. Tanuja, and K. Venugopal, "T shortest path discovery for area coverage (spdac) using prediction-based clustering in wsn," in In Advances in Artificial Intelligence and Data Engineering. Springer, 2019, pp. 1345-1357.

[17] N. Kim, J. Heo, H. S. Kim, and W. H. Kwon, "Reconfiguration of clusterheads for load balancing in wireless sensor networks," Computer Communications, vol. 31, no. 1, pp. 153-159, 2008.

[18] B. Touray, J. Shim, and P. Johnson, "Biased random algorithm for load balancing in wireless sensor networks (bralb)," in Power Electronics and Motion Control Conference (EPE/PEMC), 2012 15th International. IEEE, 2012, pp. LS4e-1.

[19] P. M. Daflapurkar, M. Gandhi, and B. Patil, "Tree based distributed clustering routing scheme for energy efficiency in wireless sensor networks," in 2017 IEEE International Conference on Power, Control, Signals and Instrumentation Engineering (ICPCSI). IEEE, 2017, pp. 2450-2456.

[20] R. C. Shah and J. M. Rabaey, "Energy aware routing for low energy ad hoc sensor networks," in Wireless Communications and Networking Conference, 2002. WCNC2002. 2002 IEEE, vol. 1. IEEE, 2002, pp. 350-355.

[21] C. Schurgers and M. B. Srivastava, "Energy efficient routing in wireless sensor networks," in Military communications conference, 2001. MILCOM 2001. Communications for network-centric operations: Creating the information force. IEEE, vol. 1. IEEE, 2001, pp. 357-361.

[22] Y. K. Yousif, R. Badlishah, N. Yaakob, and A. Amir, "An energy efficient and load balancing clustering scheme for wireless sensor network (wsn) based on distributed approach," in Journal of Physics: Conference Series, vol. 1019, no. 1. IOP Publishing, 2018, p. 012007.

[23] H. Zhang, L. Li, X.-f. Yan, and X. Li, "A load-balancing clustering algorithm of wsn for data gathering," in Artificial Intelligence, Management Science and Electronic Commerce (AIMSEC), 2011 2nd International Conference on. IEEE, 2011, pp. 915-918.

[24] Y. Liao, H. Qi, and W. Li, "Load-balanced clustering algorithm with distributed self-organization for wireless sensor networks," IEEE sensors journal, vol. 13, no. 5, pp. 1498-1506, 2013.

[25] Z. Liu, J. Zhang, Y. Li, L. Bai, and Y. Ji, "Joint jobs scheduling and light- path provisioning in fog computing micro datacenter networks," Journal of Optical Communications and Networking, vol. 10, no. 7, pp. B152-B163, 2018.

[26] T. Preethiya, A. Muthukumar, and S. Durairaj, "Double cluster head heterogeneous clustering for optimization in hybrid wireless sensor network," Wireless Personal Communications, vol. 110, no. 4, pp. 17511768, 2020.

[27] N. Zarin and A. Agarwal, "A centralized approach for load balancing in heterogeneous wireless access network," in 2018 IEEE Canadian Confer- ence on Electrical \& Computer Engineering (CCECE). IEEE, 2018, pp. 1-5.

[28] W. Yu, Y. Huang, and A. Garcia-Ortiz, "Optimal task allocation algorithms for energy constrained multihop wireless networks," IEEE Sensors Jour- nal, vol. 19, no. 17, pp. 7744-7754, 2019.

[29] T. Arvanitis, C. Constantinou, A. Stepanenko, Y. Sun, B. Liu, and K. Baughan, "Network visualisation and analysis tool based on logical network abridgment," in Military Communications Conference, 2005. MILCOM 2005. IEEE. IEEE, 2005, pp. 106-112. 
[30] K.-V. Nguyen, P. Le Nguyen, Q. H. Vu, and T. Van Do, “An energy efficient and load balanced distributed routing scheme for wireless sensor networks with holes," Journal of Systems and Software, vol. 123, pp. 92-105, 2017.

[31] X. Zhang, L. Tao, F. Yan, and D. K. Sung, "Shortest-latency opportunistic routing in asynchronous wireless sensor networks with independent duty- cycling," IEEE Transactions on Mobile Computing, 2019.

[32] A. Varga, "Omnet++ discrete event simulato," https://omnetpp.org/, 2018.
[33] Libelium "Wasp-mote agriculture boards," http://www.libelium.com/ products/waspmote/, 2018.

[34] Almaiah A, Almomani O. An investigation of digital forensics for Shamoon attack behaviour in fog computing and threat intelligence for incident response. Journal of Theoretical and Applied Information Technology. 2020 Apr 15;98(07).

[35] Almaiah A, Almomani O. An investigator of digital forensics frequencies particle swarm optimization for detection and classification of APT attack in fog computing environment (IDF-FPSO). Journal of Theoretical and Applied Information Technology. 2020 Apr 15;98(07). 